\title{
Anomalous Wave Function Statistics on a One-Dimensional Lattice with Power-Law Disorder
}

\author{
M. Titov and H. Schomerus \\ Max-Planck-Institut für Physik komplexer Systeme, Nöthnitzer Strasse 38, 01187 Dresden, Germany
}

(Received 27 February 2003; published 22 October 2003)

\begin{abstract}
Within a general framework, we discuss the wave function statistics in the Lloyd model of Anderson localization on a one-dimensional lattice with a Cauchy distribution for random on-site potential. We demonstrate that already in leading order in the disorder strength, there exists a hierarchy of anomalies in the probability distributions of the wave function, the conductance, and the local density of states, for every energy which corresponds to a rational ratio of wavelength to lattice constant. Power-law rather than log-normal tails dominate the short-distance wave-function statistics.
\end{abstract}

DOI: 10.1103/PhysRevLett.91.176601

Much of our present understanding of wave-function localization in one spatial dimension is based on the original Anderson model on a one-dimensional lattice [1]

$$
-t\left(\Psi_{n+1}+\Psi_{n-1}\right)+V_{n} \Psi_{n}=E \Psi_{n},
$$

with a white-noise disorder $\left\langle V_{n} V_{m}\right\rangle \propto \delta_{n m},\left\langle V_{n}\right\rangle=0$, and fixed hopping element $t$. The potential $V_{n}$ at each site takes real values according to a probability density $P_{V}(V)$. Most of the theoretical investigations of Anderson localization assume a finite variance $\operatorname{var} V \equiv$ $2 D<\infty$, and then consider the weak-disorder limit $D \ll t^{2}$. This condition is a prerequisite for singleparameter scaling [2], in which the ratio $L / \xi$ of propagation distance $L$ and the localization length, $\xi^{-1}=$ $-\lim _{n \rightarrow \infty} \frac{1}{n} \ln \left|\Psi_{n}\right|$, is the only free parameter in the universal distribution function of the Lyapunov exponent $\alpha=-\frac{1}{n} \ln \left|\Psi_{n}\right|$ for $n \gg \xi$. This carries over to universal distribution functions of the dimensionless conductance $g$ and the local density of states $\nu$. For weak Gaussian disorder, $D \ll t^{2}$, and energies away from the band edges and the band center, the latter is known to be distributed according to the exact log-normal law already for $L \gtrsim \lambda_{F}$ $[3,4]$, while the distribution of conductance $g$ takes a lognormal shape only asymptotically for $L \gtrsim \xi$.

In many realistic applications the distribution function $P_{V}(V)$ displays power-law tails. The most prominent example is the localization of wave functions in the momentum space of the kicked rotator [5]. This dynamical problem has been mapped onto the Anderson model with an effectively random Cauchy-distributed potential $[5,6]$

$$
P_{V, \text { Cauchy }}(V)=\pi^{-1} \operatorname{Im}[V-i \delta]^{-1}, \quad \delta>0,
$$

which has a divergent second moment, $D=\infty$, even for vanishing disorder $\delta \rightarrow 0$. Such disorder is never weak in the sense that the mean free path vanishes when calculated in second Born approximation. Equation (1) with the disorder distribution function given by Eq. (2) has been proposed for the first time by Lloyd [7]. In this model the localization length can be computed analytically for arbitrary $\delta[8,9]$, and the variance of the Lyapunov exponents $\alpha$ has been found recently in Ref. [10].
PACS numbers: 72.15.Rn, 05.45.Ac

The kicked rotator was experimentally realized in the dynamics of atoms driven by a regular train of laser pulses $[11,12]$. The probability distribution function in momentum space was seen to relax from an initial Gaussian into an exponential profile, demonstrating the absence of diffusion in momentum direction. This experiment probes the finite-distance wave function correlations and shows the exponential profile already for the shortest probed distances, where the central-limit theorem (the mechanism behind the log-normal form for $L \gg \xi$ [13]) does not apply. Since the wave-function statistics is a prototypical example of large fluctuations, it is a difficult task to relate this profile to the decay length of the typical wave function. Here, theoretical understanding of the problem has to come into play: does the profile contain any reliable information? The impact of the shape of the probability density $P_{V}(V)$ on the statistics of the wave functions has been already mentioned by Halperin [14], but has not been analyzed, let alone sufficiently appreciated, in the literature.

In this Letter we address the fluctuations of the wave function $\Psi$ for distributions $P_{V}(V)$ with power-law tails, by going beyond the mean $\xi^{-1}$ of $\alpha$ and its variance, studied so far [8-10], and having the application to the short-distance wave function statistics in mind, where the central-limit theorem [13] not yet applies. We first set out a general framework for arbitrary $P_{V}(V)$, which then is applied to the Lloyd model with $P_{V}(V)$ given by Eq. (2). The fluctuations turn out to display an anomalous energy dependence [reflecting also the spatial discreetness of the Anderson model (1)] and non-log-normal tails that strongly affect the behavior of the moments of $\Psi$, and $\nu$ even in the vanishing disorder limit $\delta \ll t$. Numerically we find that even the conductance $g$ for a finite system length $L \gtrsim \xi$ deviates distinctively from the asymptotic log-normal form resulting from the central-limit theorem, which only will be obtained for $L \gg \xi$. These characteristics of the wave-function statistics are in sharp contrast to the universality for models with $D \ll t^{2}$.

The central quantity of interest in our calculation is the generating function $\mu(\lambda)$ of the cumulants of $\ln \Psi$. As pointed out by Borland [15] and Thouless [16], instead of 
solving Eq. (1) as a boundary-value problem it suffices to investigate the specific solution $\Phi_{n}$ of the initial-value problem $\Phi_{0}=a, \Phi_{1}=b$. This solution exponentially increases as $\Phi_{n} \sim \exp (\alpha n)$ for almost all values of $a$ and $b$, which statistically is equivalent to the inverse wave-function decay $\Psi_{n} \sim \Phi_{n}^{-1}$ in the original problem. The cumulant-generating function

$$
\mu(\lambda)=\lim _{n \rightarrow \infty} \frac{1}{n} \ln \left\langle\left|\Phi_{n}\right|^{\lambda}\right\rangle=\sum_{k=1}^{\infty} \frac{c_{k}}{k !} \lambda^{k}
$$

accounts for the details of convergence of the Lyapunov exponent $\alpha$ to its mean value $c_{1}=\xi^{-1}$. The coefficients $c_{k}$ with $k \geq 2$ are numerical constants which characterize the deviations of $\alpha$ from $c_{1}$. As follows from Eq. (3) the cumulants of $\alpha$ vanish according to the law dictated by the generalized central-limit theorem $\left\langle\left\langle\alpha^{k}\right\rangle\right\rangle \sim c_{k} n^{1-k}$ for $n \gg \xi$. The coefficients $c_{k}$ do not depend on the initial conditions for $\Phi_{n}$ and capture the universal information about the fluctuations of many essential quantities in the localized regime, such as $g$ and $\nu$. The fluctuations of their logarithms can be expressed through the same coefficients $c_{k}$ by $[4,17]$

$$
\lim _{L \rightarrow \infty} L^{-1}\left\langle\left\langle(-\ln g)^{k}\right\rangle\right\rangle=L^{-1}\left\langle\left\langle(-\ln \nu)^{k}\right\rangle\right\rangle=2^{k} c_{k} .
$$

The most important consequence is that the moments $\left\langle\nu^{\lambda}\right\rangle \propto \exp [L \mu(-2 \lambda)]$ behave exponentially already for rather short distances $L$ [17], as is indeed seen in the experiments [cf. Fig. 2b of Ref. [11]]. The experimental observations now can be analyzed and understood in terms of the coefficients $c_{n}$, but this requires one to include those with $n \geq 3$. This is in contrast to the longdistance wave-function statistics, where only $c_{1}$ and $c_{2}$ are relevant quantities [13].

We now set out a general approach to calculate the generating function $\mu(\lambda)$ and the coefficients $c_{k}$ for arbitrary form of $P_{V}(V)$. We built up on the formalism previously used to calculate the inverse localization length $\xi^{-1}=c_{1}[18-20]$. The Anderson model (1) can be written in terms of new variables $z_{n}=\Psi_{n+1} / \Psi_{n}, r_{n}=$ $\ln \left|\Psi_{n}\right|$ as a map

$$
z_{n}=v_{n}-1 / z_{n-1}, \quad r_{n}=r_{n-1}+\ln \left|z_{n-1}\right|,
$$

where $v_{n} \equiv\left(V_{n}-E\right) / t$. We seek the specific solution of the initial-value problem $z_{0}=b / a, r_{0}=\ln |a|$. Iterating the map (5) we observe that $z_{n}$ and $r_{n}$ take real values $z$ and $r$ with a probability density $P_{n}(z, r)$, which obeys

$$
\begin{aligned}
P_{n}(z, r)=\int_{-\infty}^{\infty} F(v) d v \iint_{-\infty}^{\infty} & P_{n-1}\left(z^{\prime}, r^{\prime}\right) \\
& \times d z^{\prime} d r^{\prime} \delta\left(z-v+1 / z^{\prime}\right) \\
& \times \delta\left(r-r^{\prime}-\ln \left|z^{\prime}\right|\right),
\end{aligned}
$$

with $F(v)=t P_{V}(v t+E)$ the probability density of $v$.

It is convenient to introduce the function

$$
h_{n}(z, \lambda)=|z|^{\lambda} \int_{-\infty}^{\infty} e^{r \lambda} P_{n}(z, r) d r
$$

and rewrite Eq. (6) as

$$
h_{n}(z, \lambda)=|z|^{\lambda} \int_{-\infty}^{\infty} F\left(z+1 / z^{\prime}\right) h_{n-1}\left(z^{\prime}, \lambda\right) d z^{\prime} .
$$

According to Eqs. (3) and (7) we have

$$
\mu(\lambda)=\lim _{n \rightarrow \infty} \frac{1}{n} \ln \left[\int_{-\infty}^{\infty} d z h_{n-1}(z, \lambda)\right] .
$$

If the function $\mu(\lambda)$ exists, the solution to Eq. (8) at large $n$ must fulfill the relation $h_{n}(z, \lambda)=e^{\mu(\lambda)} h_{n-1}(z, \lambda)$. Thus Eq. (8) is transformed into the functional eigenvalue problem

$$
\begin{aligned}
e^{\mu(\lambda)-\lambda \ln |z|} h(z, \lambda) & =\mathcal{F}[h](z, \lambda), \\
\mathcal{F}[h](z, \lambda) & \equiv \int_{-\infty}^{\infty} F\left(z+1 / z^{\prime}\right) h\left(z^{\prime}, \lambda\right) d z^{\prime} .
\end{aligned}
$$

This is the central general equation of this Letter. In any practical case it has to be solved perturbatively in $\lambda$. We expand the function $h(z, \lambda)$ in a series $h(z, \lambda)=$ $\sum_{k=0}^{\infty} \frac{\lambda^{k}}{k !} h_{k}(z)$ and introduce the notation $\tilde{c}_{1}(z) \equiv$ $c_{1}-\ln |z|$. Equation (10) is transformed into the following set of equations:

$$
\begin{aligned}
\mathcal{F}\left[h_{0}\right]-h_{0}= & 0, \\
\mathcal{F}\left[h_{1}\right]-h_{1}= & \tilde{c}_{1} h_{0}, \\
\mathcal{F}\left[h_{2}\right]-h_{2}= & \left(\tilde{c}_{1}^{2}+c_{2}\right) h_{0}+2 \tilde{c}_{1} h_{1}, \\
\mathcal{F}\left[h_{3}\right]-h_{3}= & \left(\tilde{c}_{1}^{3}+3 \tilde{c}_{1} c_{2}+c_{3}\right) h_{0} \\
& +3\left(\tilde{c}_{1}^{2}+c_{2}\right) h_{1}+3 \tilde{c}_{1} h_{2}, \text { etc. }
\end{aligned}
$$

This hierarchy of equations determines the complete wave-function statistics for finite $n \gg \xi$. Equation (11a) delivers the stationary distribution function of $z$ and has been used before [18-20] to calculate the localization length $\xi=c_{1}^{-1}$ from

$$
c_{1}=\int_{-\infty}^{\infty} h_{0}(z) \ln |z| d z .
$$

The integrals $\int_{-\infty}^{\infty} d z$ on the left-hand side of Eqs. (11) equal zero. Equation (12) indeed can be derived by integrating both sides of Eq. (11b) along the real axis. Once the distribution function $h_{0}(z)$ and the mean Lyapunov exponent $c_{1}$ are known, one can construct the solution to Eq. (11b) iteratively by [21]

$$
\begin{aligned}
h_{1}(z) & =\mathcal{K}\left[\tilde{c}_{1} h_{0}\right](z), \\
\mathcal{K}[f](z) & \equiv-f(z)-\int_{-\infty}^{\infty} K\left(z, z^{\prime}\right) f\left(z^{\prime}\right) d z^{\prime} .
\end{aligned}
$$

The second coefficient $c_{2}$ in the cumulant expansion is found by integrating Eq. (11c) along the real axis,

$$
c_{2}=\int_{-\infty}^{\infty}\left[h_{0}(z)\left(\ln |z|-c_{1}\right)+2 h_{1}(z)\right]\left(\ln |z|-c_{1}\right) d z .
$$


This procedure can be repeated recursively to calculate all other coefficients $c_{k}$.

For the Lloyd model, the scheme developed above allows us to obtain the coefficients $c_{k}$ analytically. The stationary distribution function of the variable $z$ is found by solving the integral Eq. (11a) $[7,18]$,

$$
h_{0}(z)=\frac{1}{\pi} \operatorname{Im} \frac{1}{z-s}, \quad s+\frac{1}{s}=\frac{E+i \delta}{t}, \quad \operatorname{Im} s>0 .
$$

The integral (12) yields the well-known result $c_{1}=\ln |s|$. The kernel function $K\left(z, z^{\prime}\right)$ can be obtained by iterative application of the operator $\mathcal{F}[h]$,

$$
\begin{aligned}
K\left(z, z^{\prime}\right) & =\frac{1}{\pi} \operatorname{Im} \sum_{n=1}^{\infty}\left(\frac{1}{z-p_{n}\left(z^{\prime}\right)}-\frac{1}{z-r_{n}}\right), \\
p_{n}(z) & =\frac{\left(s^{n}-s^{-n}\right)-z\left(s^{n+1}-s^{-(n+1)}\right)}{\left(s^{n-1}-s^{-(n-1)}\right)-z\left(s^{n}-s^{-n}\right)}, \\
r_{n} & =p_{n}( \pm \infty),
\end{aligned}
$$

where the second term in the parentheses on the righthand side of Eq. (16a) is added to provide a better convergence of the intermediate expressions [21].

Applying the result (16) to Eqs. (13) and (14) and performing the summation one recovers the result of Ref. [10],

$$
\begin{aligned}
c_{2}= & \operatorname{Re}\left[\operatorname{Li}_{2}\left(\mathrm{~s}^{-2}\right)-\mathrm{Li}_{2}\left(|\mathrm{~s}|^{-2}\right)\right]+\arg (s)[\pi-\arg (s)] \\
& +\ln |s|^{2}\left[\ln \left(|s|^{2}-1\right)-\ln \left|s^{2}-1\right|\right],
\end{aligned}
$$

where $\operatorname{Li}_{n}(z)=\sum_{k=1}^{\infty} z^{k} / k^{n}$ is the polylogarithmic function. As has been shown in Ref. [10], in the limit $\delta \rightarrow 0$ the ratio $c_{2} / c_{1}$ equals 2 (not 1 as for conventional weak disorder) inside the band (it vanishes outside the band). This energy insensitivity has encouraged the authors of Ref. [10] to conclude that single-parameter scaling is fulfilled. As we will discuss now, these findings do not carry over to the fluctuations beyond the variance, characterized by $c_{k}$ with $k \geq 3$.

The coefficient $c_{3}$ can be found from Eq. (11d) as

$$
\begin{aligned}
c_{3}= & 3 \int_{-\infty}^{\infty} d z \ln \left|\frac{s}{z-1 / s}\right|\left[c_{2}-\ln ^{2}\left|\frac{s}{z}\right|\right] h_{0}(z) \\
& +3 \int_{-\infty}^{\infty} d z \ln \left|\frac{s}{z}\right|\left[\ln \left|\frac{s}{z}\right|-2 \ln \left|\frac{s}{z-1 / s}\right|\right] \Sigma(z),
\end{aligned}
$$

$\Sigma(z)=\frac{1}{2 \pi} \operatorname{Im} \sum_{n=1}^{\infty}\left[\frac{1}{z-s}-\frac{1}{z-p_{n}\left(s^{*}\right)}\right] \ln \frac{s^{*}}{p_{n}^{-1}(z)}$,

where the function $p_{n}^{-1}(z)$ stands for the inverse of $p_{n}(z)$. The ratio $c_{3} / c_{1}$ is plotted in Fig. 1 versus the energy. The plot clearly displays a sequence of sharp dips, which appear exactly at energies $E=-2 t \cos (\pi p / q)$ where $p$ and $q$ are integer, and become more narrow in the limit $\delta \rightarrow 0$. The anomaly in the band center is the biggest one and reaches about $3 \%$ of the absolute value of the ratio

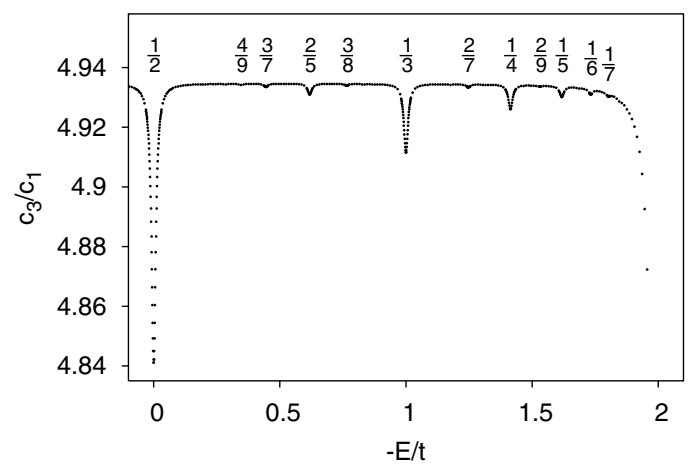

FIG. 1. The ratio $c_{3} / c_{1}$ is plotted according to the analytical result (18) for the Lloyd model at the disorder strength $\delta=$ $0.01 t$. The ratio is never small inside the band and reveals anomalies at energies $E=-2 t \cos (\pi p / q)$ with $p$ and $q$ integers. The corresponding rational number $p / q$ is indicated in the figure. The size of the anomaly depends only on the value of the denominator $q$.

$c_{3} / c_{1}$ in the limit $\delta \rightarrow 0$. The existence of such anomalies for the inverse localization length $\xi^{-1}=c_{1}$ has been pointed out by Lambert [22], but for $c_{1}$ they only show up in higher orders of the expansion in the disorder strength, with exception of the band edge $|E|=2$ and the band center $E=0[19,22,23]$. For conventional weak disorder with $D \ll t^{2}$ the other anomalies should be seen in the higher coefficients $c_{k}$ with $k \geq 3$. However, those cumulants are themselves suppressed by orders of $D / t^{2}$ (see Table I), again with the exceptions $|E|=0$, 2, where they are of the same order as $c_{1}$ and $c_{2}$ [24-26].

In contrast, in the Lloyd model the coefficients $c_{k}$ increase rapidly with increasing index $k$. In the limit $\delta \rightarrow 0$ we indeed observe $c_{2} / c_{1}=2, c_{3} / c_{1}=5, c_{4} / c_{1} \approx 20$, $c_{5} / c_{1} \approx 100$ for $p / q$ irrational. The analysis of Eqs. (11) for the Lloyd model demonstrates that the generating function $\mu(\lambda)$ exists only for $\lambda<\lambda_{c}$, where the convergence radius $\lambda_{c}<1$, which implies a factorial growth of the ratios $c_{k} / c_{1}$ for large $k$. Such a behavior is consistent with a power-law tail in the conductance distribution function $P_{g}(g) \sim g^{-\left(2-\lambda_{c}\right) / 2}$ for $g \rightarrow 0$. For a general power law $P_{V}(V) \propto|V|^{-\beta}$ for $|V| \rightarrow \infty, \quad \lambda_{c}<\beta-1$ must be expected to depend on $\beta$, implying that the precise form of the tail in $P_{g}(g)$ does depend on energy.

TABLE I. The leading asymptotic values of the coefficients $c_{k}$ in the case of weak Gaussian disorder $D \ll t^{2}$ upon the deviation $\varepsilon=2 t-|E|$ from the band edge (second column) or from the band center (third column). These results are obtained from the saddle-point analysis of Eq. (10) [24]. The last column represents the generic values inside the band.

\begin{tabular}{cccc}
\hline \hline & $D^{2 / 3} t^{-1 / 3} \ll \varepsilon \ll t$ & $D / t \ll|E| \ll t$ & $\varepsilon,|E| \sim t$ \\
\hline$c_{1}, c_{2}$ & $D /(4 t \varepsilon)$ & $D /\left(4 t^{2}\right)$ & $D /\left(4 t^{2}-E^{2}\right)$ \\
$c_{3}, c_{4}$ & $33 D^{3} /\left(128 \varepsilon^{4} t^{2}\right)$ & $9 D^{3} /\left(32 E^{2} t^{4}\right)$ & $\propto D^{3} / t^{6}$ \\
$c_{5}, c_{6}$ & $5175 D^{5} /\left(2048 \varepsilon^{7} t^{3}\right)$ & $135 D^{5} /\left(128 E^{4} t^{6}\right)$ & $\propto D^{5} / t^{10}$ \\
\hline \hline
\end{tabular}




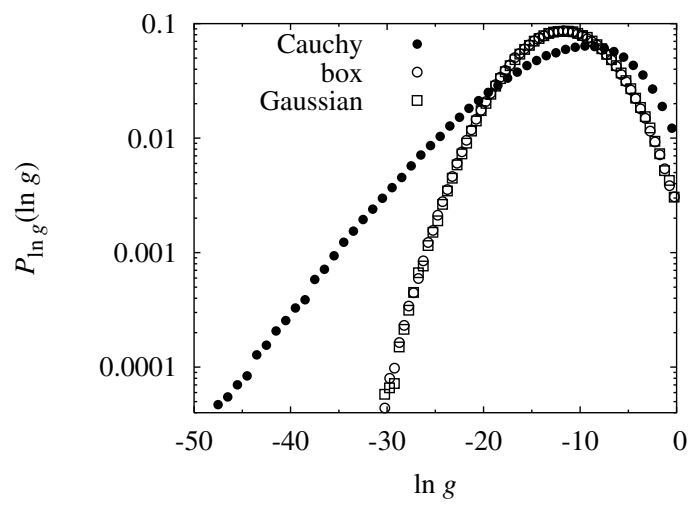

FIG. 2. Distribution function $P_{\ln g}(\ln g)$ obtained from the Anderson model (1) with Cauchy disorder (solid circles), box disorder (open circles), and Gaussian disorder (open squares). Parameters are in the localized regime $L / \xi=6$, with localization length $\xi=200$ lattice constants, and $E=0.2 t$. In this semilogarithmic plot, the log-normal distribution function for conventional weak (box or Gaussian) disorder maps to an inverted parabola, while the straight-line asymptotics found for Cauchy disorder correspond to a power-law tail in $P_{g}(g)$.

This power-law tail is observable as certified by the numerical result for Cauchy disorder and $L / \xi=6$ in Fig. 2. The large deviations from the log-normal form even at such large distances suggest that the convergence to the central-limit theorem indeed is difficult to observe in any practical experiment. The probability to find a vanishing conductance in the Lloyd model is strongly enhanced as compared to the case of conventional weak disorder, which already displays the log-normal tails. In the weak-disorder case, the generating function $\mu(\lambda)$ is finite for all $\lambda$ in the entire energy range. Moreover, far from the band edges $\left(\varepsilon \equiv 2 t-|E| \gg D^{2 / 3} t^{-1 / 3}\right)$ and far from the band center $(E \gg D / t)$, it acquires a universal parabolic form $\mu(\lambda)=\xi^{-1}\left(\lambda+\lambda^{2} / 2\right)$, since $c_{1}=c_{2}=$ $D /\left(4 t^{2}-E^{2}\right)$, while all other coefficients can be disregarded in the limit $D \ll t^{2}$ (see Table I).

In conclusion, we have studied analytically the statistics of localized wave functions in the Lloyd model, which is frequently used to analyze dynamical localization. We have found that the distribution functions of the conductance $g$ and of the local density of states $\nu$ do not have a log-normal form, up to rather large distances compared to the localization length. Moreover, even in the limit of vanishing disorder these distribution functions reveal sharp anomalies at energies $E=$ $-2 t \cos (\pi p / q)$, with $p / q$ a rational number. These features can be attributed to the power-law decay of the disorder distribution function, and sensitively affect the moments (including fractional moments) of $g$ and $\nu$ in an energy-dependent way. It is not known whether similar effects occur for the prelocalized states in the diffusive regime $L \lesssim \xi$ of multichannel systems [27].

We thank B. L. Altshuler and S. Fishman for illuminating discussions.
[1] P.W. Anderson, Phys. Rev. 109, 1492 (1958).

[2] P.W. Anderson, D. J. Thouless, E. Abrahams, and D. S. Fisher, Phys. Rev. B 22, 3519 (1980).

[3] B. L. Altshuler and V. N. Prigodin, Zh. Eksp. Teor. Fiz. 95, 348 (1989) [Sov. Phys. JETP 68, 198 (1989)].

[4] We refer here to the local density of states (regularized by opening the system), which is averaged over a range $\ell_{\text {meso }}$ such that $\lambda_{F} \ll \ell_{\text {meso }} \ll \xi$, where $\lambda_{F}$ is the Fermi wavelength. See H. Schomerus, M. Titov, P.W. Brouwer, and C.W. J. Beenakker, Phys. Rev. B 65, 121101(R) (2002).

[5] S. Fishman, D. R. Grempel, and R. E. Prange, Phys. Rev. Lett. 49, 509 (1982).

[6] D. L. Shepelyansky, Phys. Rev. Lett. 56, 677 (1986); Physica (Amsterdam) 28D, 103 (1987).

[7] P. Lloyd, J. Phys. C 2, 1717 (1969).

[8] D. J. Thouless, J. Phys. C 5, 77 (1972).

[9] K. Ishii, Prog. Theor. Phys. Suppl. 53, 77 (1973).

[10] L. I. Deych, A. A. Lisyansky, and B. L. Altshuler, Phys. Rev. Lett. 84, 2678 (2000); Phys. Rev. B 64, 224202 (2002).

[11] F. L. Moore, J. C. Robinson, C. Bharucha, P. E. Williams, and M. G. Raizen, Phys. Rev. Lett. 73, 2974 (1994).

[12] C. F. Bharucha, J.C. Robinson, F. L. Moore, B. Sundaram, Q. Niu, and M.G. Raizen, Phys. Rev. E 60, 3881 (1999).

[13] A. Cohen, Y. Roth, and B. Shapiro, Phys. Rev. B 38, 12125 (1988).

[14] B. I. Halperin, Adv. Chem. Phys. 13, 123 (1967).

[15] R. E. Borland, Proc. R. Soc. London A 274, 529 (1963).

[16] D. J. Thouless, in Ill-Condensed Matter, edited by R. Balian, R. Maynard, and G. Toulouse (NorthHolland, Amsterdam, 1979).

[17] The coefficients $c_{k}$ can be extracted from the cumulants of $\ln \nu$ already for small distances $L \gg \lambda_{F}$ to the opening. In the case of the cumulants of $\ln g$ the much stronger requirement $L \gg \xi$ has to be met, where $L$ is the system size.

[18] I. M. Lifshitz, S. A. Gredeskul, and L. A. Pastur, Introduction to the Theory of Disordered Systems (Wiley, New York, 1988).

[19] B. Derrida and E. Gardner, J. Phys. (Paris) 45, 1283 (1984).

[20] A. Bovier and A. Klein, J. Stat. Phys. 51, 501 (1988); A. Bovier, J. Stat. Phys. 56, 669 (1989).

[21] The kernel function $K\left(z, z^{\prime}\right)$ is not uniquely defined, since one can always add to it an arbitrary function depending only on $z$. The fact that such transformations do not affect the results ensures the independence of the coefficients $c_{k}$ on the conditions of the initial-value problem.

[22] C. J. Lambert, Phys. Rev. B 29, 1091 (1984).

[23] M. Kappus and F. Wegner, Z. Phys. B 45, 15 (1981).

[24] H. Schomerus and M. Titov, Phys. Rev. E 66, 066207 (2002); Phys. Rev. B 67, 100201(R) (2003).

[25] H. Schomerus and M. Titov, cond-mat/0302148.

[26] L. I. Deych, M.V. Erementchouk, and A. A. Lisyansky, Phys. Rev. Lett. 90, 126601 (2003).

[27] B. L. Altshuler, V.E. Kravtsov, and I.V. Lerner, in Mesoscopic Phenomena in Solids, edited by B. L. Altshuler, P. A. Lee, and R. A. Webb (North-Holland, Amsterdam, 1991). 